Pacific Journal of Mathematic 


\title{
THE PRIMARY DECOMPOSITION THEORY FOR MODULES
}

\author{
JoE W. FISHER
}

\begin{abstract}
Necessary and sufficient conditions are given for the classical Lasker-Noether primary decomposition theory to exist on a module over an arbitrary commutative ring. Also investigation is conducted into the connection between the existence of the primay decomposition theory and the ArtinRees property of a module.
\end{abstract}

In [1] we introduced a new technique for constructing decomposition theories for modules and we used it to give necessary and sufficient conditions for the Lesieur-Croisot tertiary decomposition theory to exist on a module over an arbitrary ring. By again making use of this technique we have obtained necessary and sufficient conditions for the classical Lasker-Noether primary decomposition theory to exist on a module over an arbitrary commutative ring. In the noncommutative case we have necessary and sufficient conditions for the primary theory to exist on an $R$-module $M$ which has the property that nil ideals are nilpotent in each factor ring of $R /(0: M)$.

In [1], [4], and [7] investigation was conducted into the connection between the existence of the primary decomposition theory and the Artin-Rees property of a module. In $\S 2$ we have obtained the following new results in this connection. For $R$ commutative we show that if an $R$-module $M$ is an Artin-Rees module and if each factor module of $M$ is finite dimensional, then $M$ has the primary decomposition theory. For $R$ noncommutative the additional hypothesis is added that nil ideals are nilpotent in each factor ring of $R /(0: M)$. In addition we show that if an $R$-module $M$ has the primary decomposition theory and if nil ideals are nilpotent in each factor ring of $R /(0: M)$, then $M$ is an Artin-Rees module.

In $\S 3$ we give some examples. In Example 1 we use our existence Theorem 1.7 to give an example of a module which has the primary decomposition theory but which is not Noetherian.

It should be brought to the attention of the reader that all the results in this paper which, for reasons of convenience, are stated for an $R$-module $M$ where $R$ is left Noetherian are also valid with the weaker assumption that nil ideals are nilpotent in each factor ring of $R /(0: M)$.

1. The primary theory. Throughout this paper, $R$ will denote 
an arbitrary associative ring which does not necessarily have an identity. All $R$-modules will be assumed to be left $R$-modules and Il will denote the category of all $R$-modules and $R$-homomorphisms.

The primary radical of an $R$-module $M$, denoted $p(M)$, is the intersection of all the prime ideals in $R$ which contain the annihilator, $(0: M)$, of $M$. A submodule $N$ of $M$ is called primary $[7,8]$ if $N \neq M$ and each $r$ in $R$, which annihilates a nonzero submodule of $M / N$, lies in $p(M / N)$. A finite set $\left\{N_{i}: i \in I\right\}$ of submodules of $M$ is a primary decomposition of $N$ in $M$ if the following conditions are satisfied:

(1) $\bigcap_{i \in I} N_{i}=N$ and for no $i \in I$ is $\bigcap_{j \neq i} N_{j} \subseteq N_{i}$;

(2) the $N_{i}, i \in I$ are primary submodules of $M$; and

(3) $p\left(M / N_{i}\right) \neq p\left(M / N_{j}\right)$ for $i \neq j$.

If each submodule of $M$ has a primary decomposition in $M$, then $M$ is said to have the primary decomposition theory.

An $R$-module $S$ is said to be $p$-stable if $S \neq(0)$ and for each nonzero submodule $N$ of $S$, then $p(N)=p(S)$. An ideal $\mathscr{P}$ in $R$ is called an associated ideal of $M$ if there exists a $p$-stable submodule $S$ of $M$ such that $\mathscr{P}=p(S)$. Denote the set of associated ideals of $M$ by $P(M)$.

Proposition 1.1. Let $M$ be an $R$-module where $R$ is either commutative or left Noetherian. If $\mathscr{P} \in P(M)$ then $\mathscr{P}$ is prime.

Proof. If $R$ is commutative the result is Proposition 9.1 in [1]. If $R$ is left Noetherian and $\mathscr{P} \in P(M)$ then there exists a $p$-stable submodule $S$ of $M$ such that $\mathscr{P}=p(S)$. Suppose that $I, J$ are ideals of $R$ such that $I J \subseteq \mathscr{P}$. Since $p(S)$ is a nil ideal modulo $(0: S)$ [3, p. 196], there exists a positive integer $n$ such that $(I J)^{n-1} S \neq(0)$ and $(I J)^{n} S=(0)$ by Levitzki's theorem [3, p. 199]. If $J \nsubseteq \mathscr{P}=p\left((I J)^{n-1} S\right)$ then $J(I J)^{n-1} S \neq(0)$. Hence $I \subseteq \mathscr{P}$ since $(I J)^{n} S=(0)$ and $\mathscr{P}=p\left(J(I J)^{n-1} S\right)$.

A submodule $N$ of $M$ is said to be a $P$-submodule if $N \neq M$ and $M / N$ is $P$-stable, i.e., there exists an ideal $\mathscr{P}$ in $R$ such that for each nonzero submodule $M^{\prime \prime}$ of $M / N, P\left(M^{\prime \prime}\right)=\{\mathscr{P}\}$. A finite set $\left\{N_{i}: i \in I\right\}$ of submodules of $M$ is a P-decomposition of $N$ in $M$ if the following conditions are satisfied:

(1) $\bigcap_{i \in I} N_{i}=N$ and for no $i \in I$ is $\bigcap_{j \neq i} N_{j} \subseteq N_{i}$;

(2) the $N_{i}, i \in I$ are $P$-submodules of $M$; and

(3) $P\left(M / N_{i}\right) \neq P\left(M / N_{j}\right)$ for $i \neq j$.

If each submodule of $M$ has a $P$-decomposition in $M$ then $M$ is said to have the P-decomposition theory. An $R$-module $M$ is called $p$-worthy if each factor module $M^{\prime \prime}$ of $M$ satisfies the following conditions: 

and

(a) each nonzero submodule of $M^{\prime \prime}$ contains a $p$-stable submodule,

(b) $P\left(M^{\prime \prime}\right)$ is finite.

In the terminology of [1] $p$ is a radical function on $\mathscr{C l}$ and $P$ is the associated ideal function on $\mathscr{C}$ that is obtained from $p$. Therefore Theorem 4.10 in [1] shows that a necessary and sufficient condition for $M$ to have the $P$-decomposition theory is that $M$ be $p$-worthy. The condition that $M$ be $p$-worthy is not a sufficient condition for $M$ to have the primary decomposition theory. See Example 4 in [1]. We proceed with the task of finding necessary and sufficient conditions for the existence of the primary theory.

LEMMA 1.2. Let $N$ be a submodule of an $R$-module $M$. Then $N$ is primary if $M / N$ is p-stable.

Proof. Suppose that $r \in R$ annihilates a nonzero submodule $M^{\prime \prime}$ of $M / N$. Then $r \in p\left(M^{\prime \prime}\right)=p(M / N)$. Hence $N$ is primary.

THEOREM 1.3. Let $R$ be an arbitrary ring and let $M$ be an $R$-module. Sufficient conditions for $M$ to have the primary decomposition theory are the following:

(1) $M$ is p-worthy, and

(2) $S$ a P-submodule of $M$ implies that $M / S$ is p-stable.

Proof. It follows from Theorem 4.10 in [1] that each submodule $N$ of $M$ has a $P$-decomposition $\left\{N_{i}: i \in I\right\}$ in $M$. Since each $N_{i}$ is a $P$-submodule of $M$, each $M / N_{i}$ is $p$-stable. Hence each $N_{i}$ is primary by Lemma 1.2. Moreover $p\left(M / N_{i}\right) \neq p\left(M / N_{j}\right)$ for $i \neq j$ because $P\left(M / N_{i}\right) \neq$ $P\left(M / N_{j}\right)$. Therefore $\left\{N_{i}: i \in I\right\}$ is a primary decomposition of $N$ in $M$.

Let $(r)$ denote the principal ideal in $R$ which is generated by $r$ in $R$.

LEMMA 1.4. Let $M$ be a nonzero $R$-module where $R$ is either commutative or left Noetherian. If $r \in p(M)$ then there exists a nonzero submodule $N$ of $M$ such that $(r) N=(0)$.

Proof. The $p(M)$ is a nil ideal modulo $(0: M)$. If $R$ is commutative then indeed $(r)$ is nilpotent modulo $(0: M)$. If $R$ is left Noetherian then $(r)$ is nilpotent modulo $(0: M)$ by Levitzki's theorem. In either case there exists a positive integer $n$ such that $(r)^{n-1} M \neq$ $(0)$ and $(r)^{n} M=(0)$. Therefore $(r)$ annihilates the nonzero submodule $(r)^{n-1} M$ of $M$.

Proposition 1.5. Let $M$ be an $R$-module where $R$ is either 
commutative or left Noetherian and let $N$ be a submodule of $M$. Then $N$ is primary if and only if $M / N$ is p-stable.

Proof. The "if" follows from 1.2.

Suppose that $N$ is primary. If $M^{\prime \prime}$ is a nonzero submodule of $M / N$ then $p(M / N) \subseteq p\left(M^{\prime \prime}\right)$. If $r \in p\left(M^{\prime \prime}\right)$ then Lemma 1.4 shows that $(r)$ annihilates a nonzero submodule of $M / N$. Wherefore $r \in p(M / N)$ since $N$ is primary. Therefore $M / N$ is $p$-stable.

Proposition 1.6. Let $M$ be an R-module where $R$ is either commutative or left Noetherian and let $N$ be a submodule of $M$. If $\left\{N_{i}: i \in I\right\}$ is a primary decomposition of $N$ in $M$ then $\left\{N_{i}: i \in I\right\}$ is a $P$-decomposition of $N$.

Proof. Proposition 1.5 yields that each $M / N_{i}$ is $p$-stable. Hence each $N_{i}$ is a $P$-submodule of $M$ because $P\left(M / N_{i}\right)=\left\{p\left(M / N_{i}\right)\right\}$. Moreover $P\left(M / N_{i}\right) \neq P\left(M / N_{j}\right)$ for $i \neq j$ since $p\left(M / N_{i}\right) \neq p\left(M / N_{j}\right)$. Therefore $\left\{N_{i}: i \in I\right\}$ is a $P$-decomposition of $N$ in $M$.

Theorem 1.7. Let $M$ be an $R$-module where $R$ is either commutative or left Noetherian. Necessary and sufficient conditions for $M$ to have the primary decomposition theory are the following:

(1) $M$ is p-worthy, and

(2) P-submodules of $M$ are primary.

Proof. The sufficiency follows from Theorem 1.3.

In order to prove the necessity assume that $M$ has the primary decomposition theory. Then $M$ has the $P$-decomposition theory by Proposition 1.6. Therefore $M$ is $p$-worthy by Theorem 4.10 in [1].

Suppose that $N$ is a $P$-submodule of $M$. Then there exists a primary decomposition $\left\{N_{i}: i=1,2, \cdots, k\right\}$ of $N$ in $M$. Moreover $\left\{N_{i}: i=1,2, \cdots, k\right\}$ is a $P$-decomposition of $N$ in $M$. Since $N$ is a $P$-submodule and $P(M / N)=\bigcup_{i=1}^{k} P\left(M / N_{i}\right)$ [1, Proposition 4.5], we have that $k=1$. Consequently $N=N_{1}$ and so $N$ is primary.

As a corollary we get the following well-known theorem.

Corollary 1.8. Let $R$ be a commutative ring and let $M$ be a Noetherian $R$-module. Then $M$ has the primary decomposition theory.

Proof. The fact that $M$ is $p$-worthy follows from Proposition 5.6 and Lemma 9.2 in [1]. That $P$-submodules of $M$ are primary follows from Proposition 9.3 in [1]. 
2. Artin-Rees modules. An $R$-module $M$ is said to be an Artin-Rees module [4, 7] if it has the Artin-Rees property, i.e., for each submodule $N$ of $M$, ideal $I$ in $R$, and positive integer $n$, there exists a positive integer $h$ such that $I^{h} M \cap N \subseteq I^{n} N$. It should be noted that submodules and factor modules of Artin-Rees modules are Artin-Rees modules. An $R$-module $M$ is said to be finite dimensional [2] over $R$ if each direct sum of nonzero submodules of $M$ has only a finite number of terms. An $R$-module $U$ is uniform if $U \neq(0)$ and each pair of nonzero submodules of $U$ has nonzero intersection. A submodule $E$ of $M$ is called essential if $E$ has nonzero intersection with each nonzero submodule of $M$.

Lemma 2.1. Let $M$ be an R-module where $R$ is either commutative or left Noetherian. If $M$ is an Artin-Rees module then $P$-submodules of $M$ are primary.

Proof. It is sufficient to show that if $(0)$ is a $P$-submodule of $M$ then (0) is primary. Suppose that $r \in R$ annihilates a nonzero submodule $N$ of $M$. Since $(0)$ is a $P$-submodule of $M$, there exists an ideal $\mathscr{P}$ in $R$ such that $P(N)=P(M)=\{\mathscr{P}\}$. Hence there exists a $p$-stable submodule $S$ of $N$ such that $\mathscr{P}=p(S)$. Whence $r \in \mathscr{P}$.

We claim that $(0:(r))=\{m \in M:(r) m=0\}$ is an essential submodule of $M$. If $W$ is a nonzero submodule of $M$ then $P(W)=\{\mathscr{P}\}$. Hence there exists a nonzero submodule $W^{\prime}$ of $W$ such that $\mathscr{P}=$ $p\left(W^{\prime}\right)$. Since $r \in \mathscr{P}$ there is a nonzero submodule $W^{\prime \prime}$ of $W^{\prime}$ such that $(r) W^{\prime \prime}=(0)$ by 1.4. Therefore $(0:(r)) \cap W \supseteqq W^{\prime \prime} \neq(0)$ and so $(0:(r))$ is essential.

Now we apply the Artin-Rees property to $(0:(r)),(r)$, and $n=1$ to obtain an $h$ such that $(r)^{h} M \cap(0:(r)) \subseteq(r)(0:(r))=(0)$. Thus $(r)^{h} M=(0)$ and so $r \in p(M)$. Therefore (0) is primary.

Theorem 2.2. Let $M$ be an $R$-module where $R$ is either commutative or left Noetherian. Sufficient conditions for $M$ to have the primary decomposition theory are the following:

(1) $M$ is p-worthy, and

(2) $M$ is an Artin-Rees module.

Proof. The result follows from Theorem 1.7 since $M$ ArtinRees guarantees that $P$-submodules of $M$ are primary.

We will now proceed to show that a module $M$ over either a commutative or left Noetherian ring has the primary decomposition theory provided that $M$ is an Artin-Rees module and each factor module of $M$ is finite dimensional. 
LEMmA 2.3. Let $M$ be an $R$-module where $R$ is either commutative or left Noetherian. If $M$ is an Artin-Rees module then uniform submodules are p-stable.

Proof. Let $U$ be a uniform submodule of $M$ and let $V$ be a nonzero submodule of $U$. Indeed $p(U) \subseteq p(V)$. Let $r \in p(V)$. By Lemma 1.4 there exists a nonzero submodule $N$ of $V$ such that $(r) N=(0)$. The Artin-Rees property of $U$ applied to $N,(r)$, and $n=1$, produces an $h$ such that $(r)^{h} U \cap N \subseteq(r) N=(0)$. Since $U$ is uniform, we have that $(r)^{h} U=(0)$. Hence $r \in p(U)$. Therefore $p(V)=p(U)$ and so $U$ is $p$-stable.

Lemma 2.4. Let $M$ be an $R$-module where $R$ is either commutative or left Noetherian. If $M$ is a finite dimensional ArtinRees module then $P(M)$ is finite.

Proof. From [2, Th. 3.3] we have that there exists an essential submodule $E$ of $M$ of the form $E=U_{1} \oplus U_{2} \oplus \cdots \oplus U_{n}$, where each $U_{i}$ is uniform. Moreover $P(M)=P(E)$ [1, Proposition 2.5] and $P(E)=\bigcup_{i=1}^{n} P\left(U_{i}\right)$ [1, Proposition 2.4]. Since uniform submodules are $p$-stable $P\left(U_{i}\right)$ consists of a single ideal. Therefore $P(M)$ is finite.

Proposition 2.5. Let $M$ be an $R$-module where $R$ is either commutative or left Noetherian. If each factor module of $M$ is finite dimensional and if $M$ is an Artin-Rees module then $M$ is p-worthy.

Proof. Let $M^{\prime \prime}$ be a factor module of $M$. Since $M^{\prime \prime}$ is a finite dimensional Artin-Rees module, each nonzero submodule of $M^{\prime \prime}$ contains a uniform [2, Lemma 3.1]; hence, $p$-stable submodule. Furthermore $P\left(M^{\prime \prime}\right)$ is finite. Therefore $M$ is $p$-worthy.

THEOREM 2.6. Let $M$ be an $R$-module where $R$ is either commutative or left Noctherian. Sufficient conditions for $M$ to have the primary decomposition theory are the following:

(1) each factor module of $M$ is finite dimensional, and

(2) $M$ is an Artin-Rees module.

Proof. The result follows immediately from Theorem 2.2 and Proposition 2.5.

Neither the hypothesis that each factor module of $M$ be finite dimensional nor the hypothesis that $M$ be an Artin-Rees module can be deleted. See Example 2 and [1, Example 4]. 
THEOREM 2.7. Let $R$ be a left Noetherian ring. If an $R$-module $M$ has the primary decomposition theory then $M$ is an Artin-Rees module.

Proof. Let $N$ be a submodule of $M, I$ an ideal in $R$, and $n$ a positive integer. Consider $I^{n} N$. Since $I^{n} \subseteq\left(I^{n} N: N\right)$, we have that $I^{n} \cong \mathscr{P}$ for each $\mathscr{P}$ in $P\left(N / I^{n} N\right)$.

Proposition 1.6 shows that $M$ has the $P$-decomposition theory. Then Proposition 11.1 in [1] produces a submodule $X$ of $M$ such that $X \cap N=I^{n} N$ and $P(M / X)=P\left(N / I^{n} N\right)$.

The submodule $X$ of $M$ has a primary decomposition $\left(M_{1}, M_{2}, \cdots\right.$, $\left.M_{k}\right\}$ in $M$ which is also a $P$-decomposition of $X$ in $M$. If $P\left\{M / M_{i}\right)=$ $\left\{\mathscr{P}_{i}\right\}$ for $i=1,2, \cdots, k$, then $P(M / X)=\left\{\mathscr{P}_{1}, \mathscr{P}_{2}, \cdots, \mathscr{P}_{k}\right\}$ by Proposition 4.5 in [1]. Because $P(M / X)=P\left(N / I^{n} N\right)$ we have that $I^{n} \subseteq \mathscr{P}_{i}$ for each $i$.

Since $M_{i}$ is a primary submodule of $M$, we have that $M / M_{i}$ is $p$-stable (1.5) and hence $\mathscr{P}_{i}=p\left(M / M_{i}\right)$ for each $i$. Whence $\mathscr{P}_{i}$ is a nil ideal modulo $\left(M_{i}: M\right)$. By Levitzki's theorem we have that for each $i$, there exists an $h_{i}$ such that $\mathscr{P}_{i}^{h_{i}} \cong\left(M_{i}: M\right)$. Accordingly there is an $h$ such that $\mathscr{P}_{i}^{h} M \subseteq M_{i}$ for each $i$. Thus $I^{n h} M \subseteq$ $M_{1} \cap M_{2} \cap \cdots \cap M_{k}=X$. So $I^{n h} M \cap N \subseteq X \cap N=I^{n} N$. Therefore $M$ is an Artin-Rees module.

As this theorem shows, a module $M$ over a left Noetherian ring which has the primary decomposition theory has the Artin-Rees property. Example 1 shows that we cannot also deduce that each factor module of $M$ is finite dimensional.

We have the following well-known theorem as a corollary.

CoRollary 2.8. Let $R$ be a commutative ring and let $M$ be an $R$-module such that $R M=M$. If $M$ is Noetherian then $M$ is an Artin-Rees module.

Proof. By using a technique similar to the one used in the proof of Theorem 2 in [6, p. 180], we can show that $R /(0: M)$ is Noetherian. Also $M$ has the primary decomposition theory by Corollary 1.8. Consequently $M$ is Artin-Rees.

The following theorem is an extension of the classical Krull "Intersection Theorem".

THEOREM 2.9. Let $R$ be a left Noetherian ring and let $M$ be an $R$-module such that

(1) $M$ is p-worthy, and 
(2) P-submodules of $M$ are primary. For an ideal $I$ of $R$, set $N=\bigcap_{n} I^{n} M$. Then $I N=N$.

Proof. Theorem 1.7 shows that $M$ has the primary decomposition theory. Hence $M$ is an Artin-Rees module by Theorem 2.7. Accordingly, there exists an $h$ such that $I^{h} M \cap N \subseteq I N$. Thus $N=$ $I^{h} M \cap N \subseteq I N \subseteq N$ and so $N=I N$.

As a corollary we get the following well-known theorem.

COROLlaRY 2.10. Let $R$ be a commutative ring and let $M$ be a Noetherian $R$-module such that $R M=M$. For an ideal $I$ of $R$, set $N=\bigcap_{n} I^{n} M$. Then $I N=N$. Furthermore, if $I$ is contained in the Jacobson radical of $R$ then $N=(0)$.

Proof. Again $R /(0: M)$ is Noetherian and $M$ has the primary decomposition theory. Therefore the result follows from 2.9.

3. Examples. The following provides an example of a module $M$ over a commutative ring which has the primary decomposition theory and is an Artin-Rees module. However there exist factor modules of $M$ which are not finite dimensional. Hence $M$ is not Noetherian.

ExAmple 1. Let $Z$ be the ring of rational integers and let $\mathscr{P}_{i}, i=1,2, \cdots, n$ be nonzero proper prime ideals in $Z$. Consider the $Z$-module $M=\sum_{j=1}^{\infty} \oplus N_{j}$ where each $N_{j}=Z / \mathscr{F}_{i}$ for some $i$. From well-known properties of semisimple modules it follows that each section, i.e., submodule of a factor module, of $M$ is isomorphic to a direct sum of a subset of $\left\{N_{j}: j=1,2, \cdots\right\}$. By making use of this fact we will show that $M$ is $p$-worthy and that $P$-submodules of $M$ are primary. Then it will follow from Theorem 1.7 that $M$ has the primary decomposition theory.

Let $M^{\prime \prime}$ be a factor module of $M$. Then $P\left(M^{\prime \prime}\right) \subseteq P(M)$. Since $P\left(Z / \mathscr{P}_{i}\right)=\left\{\mathscr{P}_{i}\right\}$, it follows from [1, Proposition 2.4] that $P(M)=$ $\mathbf{U}_{j=1}^{\infty} P\left(N_{j}\right)=\left\{\mathscr{P}_{1}, \mathscr{P}_{2}, \cdots, \mathscr{P}_{n}\right\}$. Hence $P\left(M^{\prime \prime}\right)$ is finite. That each nonzero submodule of $M^{\prime \prime}$ contains a $p$-stable submodule follows from the fact that each nonzero section of $M$ is isomorphic to a direct sum of a nonempty subset of $\left\{N_{j}: j=1,2, \cdots\right\}$. Therefore $M$ is $p$-worthy.

Suppose that $N$ is a $P$-submodule of $M$ with say $P(M / N)=\left\{\mathscr{F}_{i}\right\}$. If $z \in Z$ annihilates a nonzero submodule of $M / N$ then $z \in \mathscr{P}_{i}$. Since $P(M / N)=\left\{\mathscr{P}_{i}\right\}$ and $M / N$ is isomorphic to a direct sum of a subset of $\left\{N_{j}: j=1,2, \cdots\right\}$, we have that $M / N$ is isomorphic to a direct 
sum of copies of $Z / \mathscr{P}_{i}$. Hence $z \in p(M / N)$. Thence $N$ is primary. Consequently $M$ has the primary decomposition theory. That $M$ is an Artin-Rees module now follows from Theorem 2.7.

The following is an example of an Artin-Rees module which does not have the primary decomposition theory.

EXAMPLE 2. Let $Z$ be the ring of rational integers and let $(p)$ be the ideal in $Z$ which is generated by the prime number $p$. Consider the $Z$-module $M=\sum_{p}^{\oplus} Z /(p)$. The submodule $(0)$ does not have a primary decomposition in $M$ since $P(M)=\bigcup \mathbf{U}\{(p): p$ prime $\}$ is not finite.

We claim that $M$ is an Artin-Rees module. It is immediate that each submodule $N$ of $M$ has the form $N=\sum_{p \in S}^{\oplus} Z /(p)$ where $S \subseteq\{p: p$ prime $\}$. By using this fact we can show that for each submodule $N$ of $M$, ideal $I$ in $Z$, and positive integer $n, I^{n} M \cap N \leqq$ $I^{n} N$. Therefore $M$ is Artin-Rees.

\section{REFERENCES}

1. J. W. Fisher, Decomposition theories for modules, Trans. Amer. Math. Soc. 145 (1969), 241-269.

2. A. W. Goldie, Rings with maximum condition, Yale Univ. Lecture Notes, New Haven, Conn., 1964.

3. N. Jacobson, Structure of rings, Amer. Math. Soc. Colloq. Publ. Vol. 37, Amer. Math. Soc., Providence, R. I., 1956.

4. L. Lesieur and R. Croisot, Extension au cas non commutatif d'un théorème de Krull et d'un lemme d'Artin-Rees, J. Reine Angew. Math. 204 (1960), 216-220.

5. — Algèbre noethérienne non-commutatif, Memor. Sci. Math., Paris, 1963.

6. D. G. Northcott, Lessons on Rings, Modules, and Multiplicities, Cambridge Univ. Press, London, 1968.

7. J. A. Riley, Axiomatic primary and tertiary decomposition theory, Trans. Amer. Math. Soc. 105 (1962), 177-201.

8. O. Zariski and P. Samuel, Commutative Algebra, Vol. I, Van Nostrand, Princeton, N. J., 1958.

Received February 10, 1970.

UNIVERSITY OF TEXAS

Austin, Texas 



\section{PACIFIC JOURNAL OF MATHEMATICS}

\section{EDITORS}

\author{
H. SAMELSON \\ Stanford University \\ Stanford, California 94305

\section{Richard Pierce} \\ University of Washington \\ Seattle, Washington 98105
}

J. DugundJI

Department of Mathematics

University of Southern California

Los Angeles, California 90007

RichaRd ARENS

University of California

Los Angeles, California 90024

\section{ASSOCIATE EDITORS}
E. F. BECKENBACH
B. H. NeUMANN
F. WOLE
K. YoshidA

\section{SUPPORTING INSTITUTIONS}

\author{
UNIVERSITY OF BRITISH COLUMBIA \\ CALIFORNIA INSTITUTE OF TECHNOLOGY \\ UNIVERSITY OF CALIFORNIA \\ MONTANA STATE UNIVERSITY \\ UNIVERSITY OF NEVADA \\ NEW MEXICO STATE UNIVERSITY \\ OREGON STATE UNIVERSITY \\ UNIVERSITY OF OREGON \\ OSAKA UNIVERSITY \\ UNIVERSITY OF SOUTHERN CALIFORNIA
}

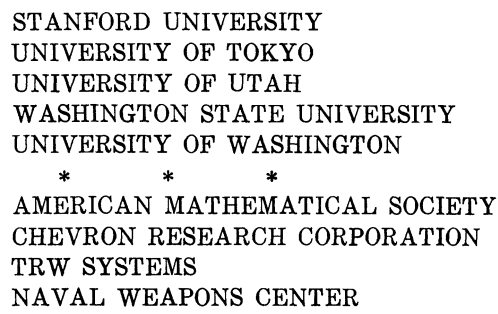

The Supporting Institutions listed above contribute to the cost of publication of this Journal, but they are not owners or publishers and have no responsibility for its content or policies.

Mathematical papers intended for publication in the Pacific Journal of Mathematics should be in typed form or offset-reproduced, (not dittoed), double spaced with large margins. Underline Greek letters in red, German in green, and script in blue. The first paragraph or two must be capable of being used separately as a synopsis of the entire paper. The editorial "we" must not be used in the synopsis, and items of the bibliography should not be cited there unless absolutely necessary, in which case they must be identified by author and Journal, rather than by item number. Manuscripts, in duplicate if possible, may be sent to any one of the four editors. Please classify according to the scheme of Math. Rev. Index to Vol. 39. All other communications to the editors should be addressed to the managing editor, Richard Arens, University of California, Los Angeles, California, 90024.

50 reprints are provided free for each article; additional copies may be obtained at cost in multiples of 50 .

The Pacific Journal of Mathematics is published monthly. Effective with Volume 16 the price per volume (3 numbers) is $\$ 8.00$; single issues, $\$ 3.00$. Special price for current issues to individual faculty members of supporting institutions and to individual members of the American Mathematical Society: $\$ 4.00$ per volume; single issues $\$ 1.50$. Back numbers are available.

Subscriptions, orders for back numbers, and changes of address should be sent to Pacific Journal of Mathematics, 103 Highland Boulevard, Berkeley, California, 94708.

PUBLISHED BY PACIFIC JOURNAL OF MATHEMATICS, A NON-PROFIT CORPORATION

Printed at Kokusai Bunken Insatsusha (International Academic Printing Co., Ltd.), 7-17, Fuj̣imi 2-chome, Chiyoda-ku, Tokyo, Japan. 


\section{Pacific Journal of Mathematics}

\section{Vol. 35, No. $2 \quad$ October, 1970}

Valentin Danilovich Belousov and Palaniappan L. Kannappan, Generalized Bol functional equation .................................... 259

Charles Morgan Biles, Gelfand and Wallman-type compactifications ........... 267

Louis Harvey Blake, A generalization of martingales and two consequent convergence theorems .................................... 279

Dennis K. Burke, On p-spaces and $w \Delta$-spaces..................... 285

John Ben Butler, Jr., Almost smooth perturbations of self-adjoint operators . . . . . . 297

Michael James Cambern, Isomorphisms of $C_{0}(Y)$ onto $C(X) \ldots \ldots \ldots \ldots \ldots . \ldots 307$

David Edwin Cook, A conditionally compact point set with noncompact closure ... 313

Timothy Edwin Cramer, Countable Boolean algebras as subalgebras and homomorphs .........................................

John R. Edwards and Stanley G. Wayment, A v-integral representation for linear operators on spaces of continuous functions with values in topological vector spaces.............................................

Mary Rodriguez Embry, Similarities involving normal operators on Hilbert

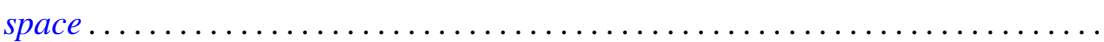

Lynn Harry Erbe, Oscillation theorems for second order linear differential

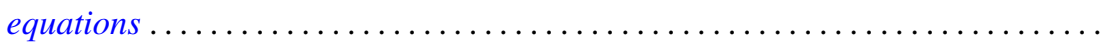

William James Firey, Local behaviour of area functions of convex bodies .......... Joe Wayne Fisher, The primary decomposition theory for modules ..............

Gerald Seymour Garfinkel, Generic splitting algebras for Pic ..................

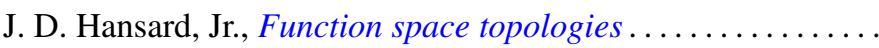

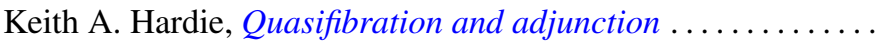

G. Hochschild, Coverings of pro-affine algebraic groups ...........

Gerald L. Itzkowitz, On nets of contractive maps in uniform spaces ..

381

389

399

417

Melven Robert Krom and Myren Laurance Krom, Groups with free nonabelian subgroups....................................

James Robert Kuttler, Upper and lower bounds for eigenvalues by finite differences ......................................

Dany Leviatan, A new approach to representation theory for convolution transforms . . .

Richard Beech Mansfield, Perfect subsets of definable sets of real numbers ...

Brenda MacGibbon, A necessary and sufficient condition for the embedding of a

Lindelof space in a Hausdorff $\mathscr{H} \sigma$ space ..................

David G. Mead and B. D. McLemore, Ritt's question on the Wronskian ....

Edward Yoshio Mikami, Focal points in a control problem .....

Paul G. Miller, Characterizing the distributions of three independent n-dimensional random variables, $X_{1}, X_{2}, X_{3}$, having analytic characteristic functions by the joint distribution of $\left(X_{1}+X_{3}, X_{2}+X_{3}\right)$. . .

P. Rosenthal, On the Bergman integral operator for an elliptic partial differential equation with a singular coefficient....

Douglas B. Smith, On the number of finitely generated $O$-group 